
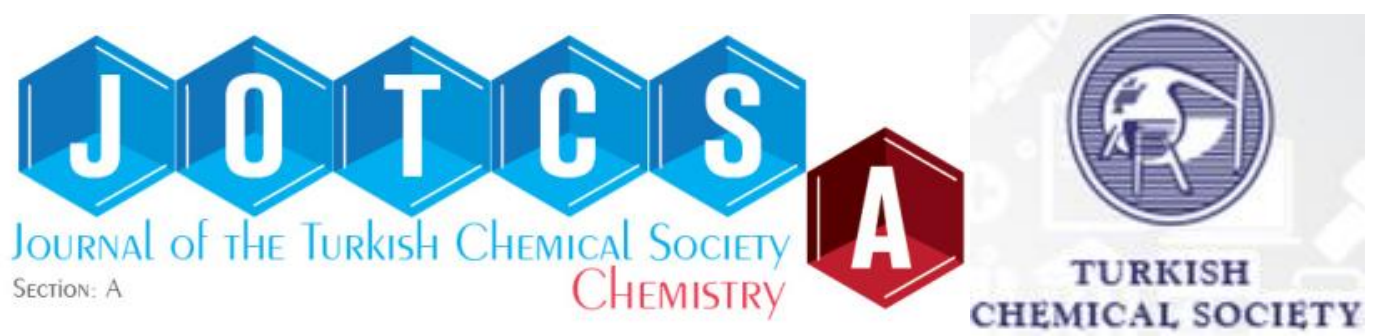

\title{
Syntheses and characterization of new dithiophosphinato zinc complexes
}

\author{
Ertuğrul Gazi Sağlam1 $\square$ and Nurcan Acar ${ }^{2 *} \square$ \\ ${ }^{1}$ Department of Chemistry, Bozok University, 66900, Yozgat, Turkey, \\ 2 Department of Chemistry, Ankara University, 06100 Tandoğan, Ankara, Turkey
}

Abstract : Four known dithiophosphinic acids, DTPA, were synthesized and their zinc complexes were newly prepared. The complexes have been proven to have the structures $\left[\mathrm{Zn}\left(\mu-\mathrm{DTPA}^{\mathrm{n}}\right)_{2}\left(\mathrm{DTPA}^{n}\right)_{2}\right]$, $\left(\left[D_{T P A}{ }^{n}\right]=\mathrm{R}^{\mathrm{n}}(\mathrm{R}) \mathrm{P}(\mathrm{S}) \mathrm{H}, \mathrm{R}=4-\mathrm{CH}_{3} \mathrm{O}-\mathrm{C}_{6} \mathrm{H}_{4}{ }^{-} ; \mathrm{R}^{1}=\right.$ iso-amyl-, $\mathrm{DTPA}^{1} ; \mathrm{R}^{2}=$ iso-butyl-, $\mathrm{DTPA}^{2} ; \mathrm{R}^{3}=$ sec-butyl-, $\mathrm{DTPA}^{3} ; \mathrm{R}^{4}=$ iso-propyl-, DTPA ${ }^{4}$. The DTPAs were prepared by the reaction of the Lawesson reagent, [2,4-bis(4-methoxyphenyl)-1,3,2,4-dithiodiphosphetane-2,4-disulfide] (LR), with the corresponding Grignard compounds. The acids formed are viscous liquids difficult to purify and so they were converted to ammonium salts, $\left[D T P A^{n}\right]\left[\mathrm{NH}_{4}\right]$, which are easy to obtain as pure crystals. The complexes were identified to have dimeric structures on the basis of mass spectroscopic data.

Keywords: Dithiophosphinic Acid, Dithiophosphinate, Phosphinodithioic Acid, Dithiophosphinato Zinc Complex, Lawesson's Reagent.

Submitted: May 25, 2018. Accepted: .July 30, 2018

Cite this: Sağlam E, Acar N. Syntheses and characterization of new dithiophosphinato zinc complexes. JOTCSA. 2018;5(2):931-40.

DOI: http://dx.doi.org/10.18596/jotcsa.427192.

*Corresponding author. E-mail: nurcanacar97@gmail.com

\section{INTRODUCTION}

Acidic organodithiophosphorus compounds like dithiophosphoric, dithiophosphonic and dithiophosphinic acids (Figure 1) and the coordination compounds thereof have some industrial interest because of their use as a rubber vulcanizing agent $(1,2)$, lubricating oil additives (3-5), metal flotation mediators (6-8), and pesticides (9-11). Potential uses as chemotherapeutic agents (12), clinical imaging aids (13) and antibiotic synergists are also investigated (14).<smiles>OP(O)(=S)S</smiles>

Dithiophosphoric acid<smiles>[R]P(=S)(S)S</smiles>

Dithiophosphonic acid $\mathrm{R}, \mathrm{R}^{\prime}=$ Alkyl- or aryl- groups<smiles>[R][PH]([R])(=S)=S</smiles>

Dithiophosphinic acid

Figure 1. Different types of organodithiophosphorus compounds

DTPA metal complexes are mostly classified as four-coordination number. The nickel(II)-DTPA complexes are mono-nucleic and as a squareplanar (15), on the other hand $\mathrm{Zn}(\mathrm{II})$ - or Cd(II)DTPA complexes are shown to be four coordinated, dimeric structures $(16,17)$. Here, we present the preparation and characterization of the zinc complexes of four different dithiophosphinic acids, [DTPA ${ }^{n}$, 4methoxyphenyl(3-methylbutyl) dithiophosphinic acid, [DTPA ${ }^{1}$; 4 4-methoxyphenyl(2methylpropyl) dithiophosphihinic acid, [DTPA ${ }^{2}$; 
4-methoxyphenyl(1-methylpropyl)

dithiophosphinic acid, [DTPA ${ }^{3}$; methoxyphenyl(1-methylpropyl)

dithiophosphinic acid, [DTPA ${ }^{4}$ ]. The synthesis of the dithiophosphinato ligand was described elsewhere (18-20). As it is general for the related compounds the Lawesson reagent, 2,4bis(4-methoxyphenyl)-1,3,2,4-

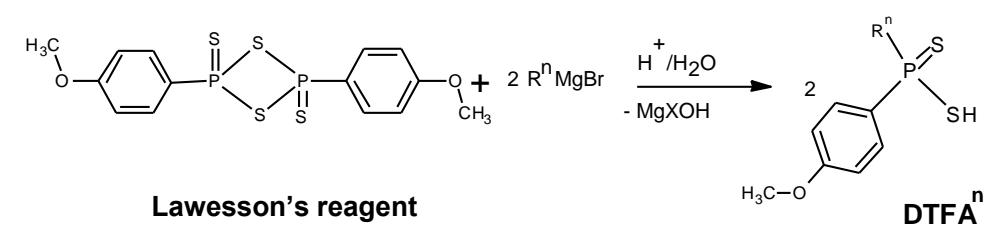<smiles>COc1ccc(P(=S)(S)[SnH2]N)cc1</smiles>

DTFA $^{n}$

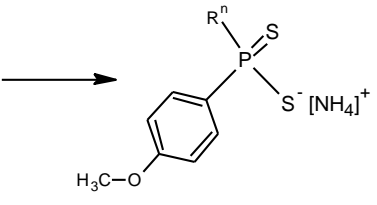

[DTFA ] $\left[\mathrm{NH}_{4}\right.$ ] dithiadiphosphetane-2,4-disulfide (LR) was reacted with the appropriate Grignard reagent to obtain the corresponding dithiophosphinic acid and the product was neutralized with dry ammonia to yield a crystallizable ammonium salt for purification, [DTPA $\left.{ }^{\mathrm{n}}\right]\left[\mathrm{NH}_{4}\right]$. The reactions and the products are summarized in Scheme 1.

Scheme 1. Synthesis of [DTPA $\left.{ }^{\mathrm{n}}\right]$ and $\left[\mathrm{DTPA}^{\mathrm{n}}\right]\left[\mathrm{NH}_{4}\right]$.
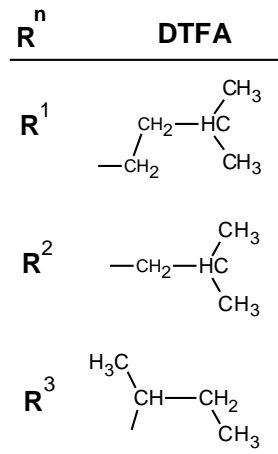

$\mathbf{R}^{4}$

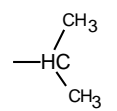

Zinc complexes of the four dithiophosphinic acids described above were obtained by the treatment of the corresponding ammonium-

$\left[\right.$ DTFA $\left.^{\mathrm{n}}\right]\left[\mathrm{NH}_{4}\right]$

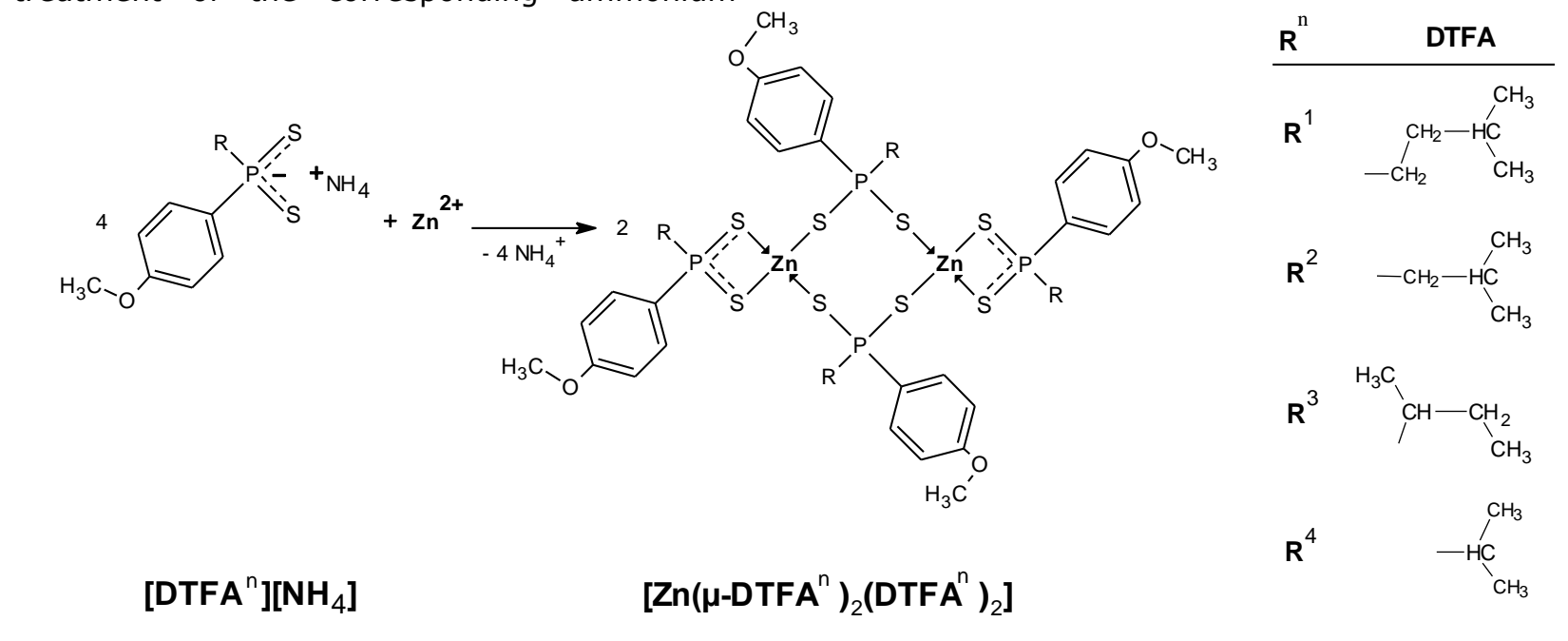

ligand salt with zinc(II)chloride in ethanolic medium, $\left[\mathrm{Zn}\left(\mu-\mathrm{DTPA}^{\mathrm{n}}\right)_{2}\left(\mathrm{DTPA}^{\mathrm{n}}\right)_{2}\right]$, (Scheme 2$)$.

Scheme 2. Synthesis of $\left[Z n\left(\mu-D_{T P A}^{n}\right)_{2}\left(D_{T P A}^{n}\right)_{2}\right]$.

\section{EXPERIMENTAL}

\section{General}

Analytical-grade LR, iso-amyl bromide, iso-butyl bromide, sec-butyl bromide and iso-propyl bromide were purchased from Merck and used directly without further purification. Benzene, chloroform, ethanol, diethyl ether and $\mathrm{ZnCl}_{2}$ were purchased from Merck. Benzene and diethyl ether were distilled and dried before use. $\left[\mathrm{DTPA}^{1}\right]\left[\mathrm{NH}_{4}\right],\left[\mathrm{DTPA}^{2}\right]\left[\mathrm{NH}_{4}\right],\left[\mathrm{DTPA}^{3}\right]\left[\mathrm{NH}_{4}\right]$ and $\left[\mathrm{DTPA}^{4}\right]\left[\mathrm{NH}_{4}\right]$ were prepared according to the literature (21).

The LC/MS system was supplied by Waters with a C-18 HPLC column and a Waters Micromass ZQ connected to an ESI ionizer. Melting point determinations were performed on a Gallenkamp apparatus with a glass capillary. ${ }^{1} \mathrm{H}$, protoncoupled ${ }^{13} \mathrm{C}$ and ${ }^{31} \mathrm{P}$ NMR spectra were recorded on a Varian Mercury (Agilent) $400 \mathrm{MHz}$ FT spectrometer. $\mathrm{CDCl}_{3}$ was the solvent of preferences for zinc complexes. Internal standards were $\mathrm{SiMe}_{4}$ for the ${ }^{1} \mathrm{H}-,{ }^{13} \mathrm{C}-\mathrm{NMR}$ and $85 \% \mathrm{H}_{3} \mathrm{PO}_{4}$ for ${ }^{31} \mathrm{P}-\mathrm{NMR}$. IR spectra were recorded on a Perkin Elmer Spectrum 400 FTIR instrument $\left(200-4000 \mathrm{~cm}^{-1}\right)$ with wavenumbers in $\mathrm{cm}^{-1}$ units. Elemental analyses were performed on a LECO CHNS-932 C instrument.

\section{General procedure for the zinc complexes}

To pure ethyl alcoholic solution of $2 \mathrm{mmol}(0.04$ g) of $\mathrm{ZnCl}_{2}$ in a beaker $(40-50 \mathrm{~mL})$ were added 4 mmoles of the ligand of interest $(0.34 \mathrm{~g}$ of 
$\left[\mathrm{DTFA}^{1}\right]\left[\mathrm{NH}_{4}\right] ; 0.31 \mathrm{~g}$ of $\left[\mathrm{DTFA}^{2}\right]\left[\mathrm{NH}_{4}\right] ; 0.31 \mathrm{~g}$ of $\left[\mathrm{DTFA}^{3}\right]\left[\mathrm{NH}_{4}\right]$ and $0.28 \mathrm{~g}$ of $\left[\mathrm{DTFA}^{4}\right]\left[\mathrm{NH}_{4}\right] ;$ ). After mixing the cation with the ligand, the colorless complex was formed. Upon waiting for 2 days, fine crystals of the complex were formed at the bottom. These crystals were filtered and recrystallized from ethanol or chloroform.

Bis-\{bis-[4-methoxyphenyl(3-

methylbutyl)dithiophosphinato]zinc(II) $\}$

$\left[\mathrm{Zn}\left(\mu-\mathrm{DTPA}^{1}\right)_{2}\left(\mathrm{DTPA}^{1}\right)_{2}\right]$. Yield: $1.83 \mathrm{~g}, 74 \%$. Colorless. M.P. $221-222^{\circ} \mathrm{C} .{ }^{1} \mathrm{H}$ NMR ( $\delta: \mathrm{ppm}$, $\left.\mathrm{CDCl}_{3}\right): \delta=0.85\left(\mathrm{dd}, 3_{\mathrm{HH}}=6.51 \mathrm{~Hz}, 24 \mathrm{H},-\mathrm{CH}-\right.$ $\left.\left(\mathrm{CH}_{3}\right)_{2}\right), 1.47(\mathrm{~m}, 8 \mathrm{H},-\mathrm{C} \underline{\mathrm{H}}-), 1.55\left(\mathrm{~m}, 4 \mathrm{H},-\mathrm{CH}_{2}-\right.$ $\mathrm{CH}), 2.28\left(\mathrm{~m}, 8 \mathrm{H},-\mathrm{CH}_{2}-\mathrm{CH}_{2}\right), 3.84(\mathrm{~s}, 12 \mathrm{H}$, $\left.\mathrm{OC}_{\mathrm{H}_{3}}\right), 6.95\left(\mathrm{~A}\right.$-part of $\mathrm{AA}^{\prime} \mathrm{MM}^{\prime} \mathrm{X},{ }^{4} \mathrm{~J}_{\mathrm{PH}}=2.30 \mathrm{~Hz}$ $\left.\left(J_{\mathrm{AX}}\right), N=8.70 \mathrm{~Hz}, 8 \mathrm{H}, m-\mathrm{H}\right), 7.86 \mathrm{M}$-part of $\mathrm{AA}^{\prime} \mathrm{MM}^{\prime} \mathrm{X},{ }^{3} \mathrm{~J}_{\mathrm{PH}}=13.36 \mathrm{~Hz}\left(J_{\mathrm{MX}}\right), N=8.70 \mathrm{~Hz}, 8 \mathrm{H}$, $0-\mathrm{H}) .{ }^{13} \mathrm{C}-\mathrm{NMR}\left(\mathrm{CDCl}_{3}\right): \delta=22.1\left(\mathrm{~s},-\left(\mathrm{CH}_{3}\right)_{2}\right)$, $\left.32.0\left(\mathrm{~d},{ }^{3} \mathrm{~J}_{\mathrm{P}-\mathrm{C}}=4.4 \mathrm{~Hz},-\underline{\mathrm{CH}}-\left(\mathrm{CH}_{3}\right)_{2}\right)\right), 28.6\left(\mathrm{~d},{ }^{2} \mathrm{~J}_{\mathrm{P}-}\right.$ $\left.\mathrm{c}=17.5 \mathrm{~Hz},-\underline{\mathrm{H}}_{2}-\mathrm{CH}\right), 40.2\left(\mathrm{~d}, \mathrm{~J}_{\mathrm{P}-\mathrm{C}}=50.9 \mathrm{~Hz}, \mathrm{P}-\right.$ $\left.\mathrm{C}_{2}{ }^{-}\right), 55.4$ (s, $\left.\underline{\mathrm{C}}_{3} \mathrm{O}-\right), 113.3\left(\mathrm{~d},{ }^{3} \mathrm{~J}_{\mathrm{P}-\mathrm{C}}=14.2 \mathrm{~Hz}\right.$, Ar-C $\left.{ }_{\text {meta }}\right), 126.5$ (d, $J_{\mathrm{P}-\mathrm{C}}=88.9 \mathrm{~Hz}, \mathrm{P}-\mathrm{C}_{\text {arom }}$ ) $132.3\left(\mathrm{~d},{ }^{2} \mathrm{~J}_{\mathrm{P}-\mathrm{C}}=11.9 \mathrm{~Hz}\right.$, Ar-Cortho $), 162.1$ (s, $\left.\mathrm{CH}_{3} \mathrm{O}-\underline{\mathrm{C}}\right)$. ${ }^{31 \mathrm{P}-\mathrm{NMR}}\left(\mathrm{CdCl}_{3}\right): \delta=68.1$. LC/MS: $\mathrm{m} / \mathrm{z}$ $647.3\left(\left[\left[\mathrm{M} / 2+\mathrm{CH}_{3} \mathrm{CN}\right)\right]^{+}, 100 \%\right), 1222.9\left([\mathrm{M}]^{+}\right.$, $4 \%), 883.3\left(\left[\mathrm{M}-\mathrm{DTPA}^{1}\right]^{+}, 4 \%\right), 614.3\left(\left[\mathrm{M} / 2-\mathrm{H}^{+}{ }^{+}\right.\right.$, $6 \%)$. Anal. Calcd. for $\mathrm{C}_{48} \mathrm{H}_{72} \mathrm{Zn}_{2} \mathrm{O}_{4} \mathrm{P}_{4} \mathrm{~S}_{8}$ (1224.3 g. $\mathrm{mol}^{-1}$ ): $\mathrm{C}, 47.1 ; \mathrm{H}, 5.9 ; \mathrm{S}, 21.0$. Found: C, $47.2 ; \mathrm{H}, 5.7 ; \mathrm{S}, 20.8 \%$.

Bis-\{bis-[4-methoxyphenyl(2-

methylpropyl)dithiophosphinato]zinc(II)\}

$\left[\mathrm{Zn}\left(\mu-\mathrm{DTPA}^{2}\right)_{2}\left(\mathrm{DTPA}^{2}\right)_{2}\right]$. Yield: $2.54 \mathrm{~g}, 85 \%$. Colorless. M.P. $167-168^{\circ} \mathrm{C}$. ${ }^{1} \mathrm{H}$ NMR $\left(\mathrm{CDCl}_{3}\right): \delta=$ $0.88\left(\mathrm{t},{ }^{3} \mathrm{~J}_{\mathrm{HH}}=6.67 \mathrm{~Hz}, 24 \mathrm{H},-\mathrm{CH}_{2}-\mathrm{CH}_{3}\right), 2.11$ $\left(\mathrm{m}, 4 \mathrm{H},-\mathrm{CH}-\mathrm{CH}_{3}\right), 2.28\left(\mathrm{~m}, 8 \mathrm{H},{ }^{2} \mathrm{~J}_{\mathrm{PH}}=11.90 \mathrm{~Hz}\right.$, $\left.{ }^{3} J_{\mathrm{PH}}=6.10 \mathrm{~Hz} \mathrm{P}-\underline{\mathrm{C}}_{2}\right), 3.84\left(\mathrm{~s}, 12 \mathrm{H}, \mathrm{OC}_{3}\right), 6.92$ (A-part of $\mathrm{AA}^{\prime} \mathrm{MM}^{\prime} \bar{X},{ }^{4} \mathrm{~J}_{\mathrm{PH}}=2.46 \mathrm{~Hz}\left(J_{\mathrm{AX}}\right), N=8.50$ $\mathrm{Hz}, 8 \mathrm{H}, m-\mathrm{H}), 7.91 \mathrm{M}$-part of $A A^{\prime} M M^{\prime} X,{ }^{3} J_{\mathrm{PH}}=$ $\left.13.44 \mathrm{~Hz}\left(J_{\mathrm{MX}}\right), N=8.50 \mathrm{~Hz}, 8 \mathrm{H}, O-\mathrm{H}\right) .{ }^{13} \mathrm{C}-\mathrm{NMR}$ $\left(\mathrm{CDCl}_{3}\right): \delta=25.5\left(\mathrm{~d},{ }^{3} \mathrm{~J}_{\mathrm{P}-\mathrm{C}}=3.9 \mathrm{~Hz},-\left(\mathrm{CH}_{3}\right)_{2}\right)$, $\left.24.3\left(\mathrm{~d},{ }^{2} \mathrm{~J}_{\mathrm{P}-\mathrm{C}}=10.0 \mathrm{~Hz},-\underline{\mathrm{C}} \mathrm{H}-\left(\mathrm{CH}_{3}\right)_{2}\right)\right), 42.9(\mathrm{~d}$, $\left.J_{\mathrm{P}-\mathrm{C}}=51.4 \mathrm{~Hz}, \mathrm{P}-\mathrm{CH}_{2}-\right), 55.4\left(\mathrm{~s}, \underline{\mathrm{CH}}_{3} \mathrm{O}-\right), 113.7$ $\left(\mathrm{d},{ }^{3} \mathrm{~J}_{\mathrm{P}-\mathrm{C}}=14.1 \mathrm{~Hz}, \mathrm{Ar}-\mathrm{C}_{\text {meta }}\right), 127.8\left(\mathrm{~d}, \mathrm{~J}_{\mathrm{P}-\mathrm{C}}=\right.$ $\left.80.3 \mathrm{~Hz}, \mathrm{P}-\mathrm{C}_{\text {arom }}\right) 132.6$ (d, $2 \mathrm{~J}_{\mathrm{P}-\mathrm{C}}=13.1 \mathrm{~Hz}$, ArCortho), 161.9 (d, $\left.{ }^{4} J_{\mathrm{P}-\mathrm{C}}=3.0 \mathrm{~Hz}, \mathrm{CH}_{3} \mathrm{O}-\mathrm{C}\right)$. ${ }^{31} \mathrm{P}-$ NMR $\left(\mathrm{CDCl}_{3}\right): \delta=60,7$. LC/MS: $m / z \quad 1190.3$ $\left([\mathrm{M}+\mathrm{Na}]^{+}, 2 \%\right), 845.2\left(\left[\mathrm{M}-\left(\mathrm{Zn}\left(\mathrm{DTPA}^{2}\right)\right]^{+}, 4 \%\right)\right.$, $619.3\left(\left[\mathrm{M} / 2+\mathrm{CH}_{3} \mathrm{CN}\right]^{+}, \quad 92 \%\right), \quad 607.2$ $\left([\mathrm{M} / 2+\mathrm{Na}]^{+}\right.$, 35\%). Anal. Calcd. for $\mathrm{C}_{44} \mathrm{H}_{64} \mathrm{Zn}_{2} \mathrm{O}_{4} \mathrm{P}_{4} \mathrm{~S}_{8}\left(1168.2 \mathrm{~g} \mathrm{~mol}^{-1}\right): \mathrm{C}, 45.2 ; \mathrm{H}$, 5.5; S, 22.0. Found: C, 45.4; H, 5.5; S, $22.2 \%$.

Bis- $\{$ bis-[4-methoxyphenyl(1methylpropyl)dithiophosphinato]zinc(II)\} $\left[\mathrm{Zn}\left(\mu-\mathrm{DTPA}^{3}\right)_{2}\left(\mathrm{DTPA}^{3}\right)_{2}\right]$. Yield: $2.33 \mathrm{~g}, 78 \%$. Colorless. M.P. $187-188^{\circ} \mathrm{C}$. ${ }^{1} \mathrm{H}$ NMR $\left(\mathrm{CDCl}_{3}\right): \delta=$ $0.92\left(\mathrm{t},{ }^{3} \mathrm{~J}_{\mathrm{HH}}=7.30 \mathrm{~Hz}, 12 \mathrm{H},-\mathrm{CH}_{2}-\mathrm{CH}_{3}\right), 1.98$ $\left(\mathrm{m}, 8 \mathrm{H},-\mathrm{C}_{2}-\mathrm{CH}_{3}\right), 1.16\left(\mathrm{~m},-\mathrm{CH}_{3}-\mathrm{CH}\right.$ - adjacent with $-\mathrm{CH}_{3}-\mathrm{CH}-16 \mathrm{H}$ ), 1.16 (dd, $-\mathrm{CH}_{3}-\mathrm{CH}-$ ve $\mathrm{CH}_{3}-\mathrm{CH}$ - adjacent, ${ }^{2} \mathrm{~J}_{\mathrm{PH}}=21.94 \mathrm{~Hz},{ }^{3} \mathrm{~J}_{\mathrm{PH}}=6.86$
$\mathrm{Hz}, 16 \mathrm{H}), 3.84\left(\mathrm{~s}, 12 \mathrm{H}, \mathrm{OCH}_{3}\right), 6.95$ (A-part of $\mathrm{AA}^{\prime} \mathrm{MM}^{\prime} \mathrm{X},{ }^{4} \mathrm{~J}_{\mathrm{PH}}=2.32 \mathrm{~Hz}\left(J_{\mathrm{AX}}\right), N=8.80 \mathrm{~Hz}, 8 \mathrm{H}$, $m-\mathrm{H}), 7.83 \mathrm{M}$-part of $\mathrm{AA}^{\prime} \mathrm{MM}^{\prime} \mathrm{X},{ }^{3} \mathrm{~J}_{\mathrm{PH}}=12.95 \mathrm{~Hz}$ $\left.\left(J_{\mathrm{MX}}\right), N=8.80 \mathrm{~Hz}, 4 \mathrm{H}, O-\mathrm{H}\right) .{ }^{13} \mathrm{C}-\mathrm{NMR}$ (ppm, $\left.\left.\mathrm{CDCl}_{3}\right): \delta=16.26\left(\mathrm{~d},{ }^{2}\right]_{\mathrm{P}-\mathrm{C}}=1.0 \mathrm{~Hz},-\left(\mathrm{CH}_{3}\right)_{2}\right)$, $38.19\left(\mathrm{~d}, \mathrm{~J}_{\mathrm{P}-\mathrm{C}}=49.6 \mathrm{~Hz}, \mathrm{P}-\underline{\mathrm{C}} \mathrm{H}-\right), 55.37$ (s, $\left.\mathrm{CH}_{3} \mathrm{O}-\right), 113.6\left(\mathrm{~d},{ }^{3} \mathrm{~J}_{\mathrm{p}-\mathrm{C}}=13.9 \mathrm{~Hz}, \mathrm{Ar}-\mathrm{C}_{\text {meta }}\right), \delta=$ $124.85\left(\mathrm{~d}, \mathrm{~J}_{\mathrm{P}-\mathrm{C}}=78.1 \mathrm{~Hz}, \mathrm{P}-\mathrm{C}_{\text {arom }}\right) 132.97$ (d, ${ }^{2} \mathrm{~J}_{\mathrm{P}-\mathrm{C}}=12.5 \mathrm{~Hz}$, Ar-C ortho $), 162.05\left(\mathrm{~d},{ }^{4} \mathrm{~J}_{\mathrm{P}-\mathrm{C}}=2.8\right.$ $\left.\mathrm{Hz}, \mathrm{CH}_{3} \mathrm{O}-\underline{\mathrm{C}}\right)$. ${ }^{31} \mathrm{P}-\mathrm{NMR}\left(\mathrm{ppm}, \mathrm{CDCl}_{3}\right): \delta=82.9$. LC/MS: $m / z$ 1191.1 $\left([\mathrm{M}+\mathrm{Na}]^{+}, 31.2 \%\right) ; 909.1$ $\left(\left[\left(\mathrm{M}-\left(\mathrm{Zn}\left(\mathrm{DTPA}^{3}\right)\right]^{+}, 89.0 \%\right) ; 583.2\left([\mathrm{M} / 2-\mathrm{H}]^{+}\right.\right.\right.$, $78,5 \%)$. Anal. Calcd. for $\mathrm{C}_{44} \mathrm{H}_{64} \mathrm{Zn}_{2} \mathrm{O}_{4} \mathrm{P}_{4} \mathrm{~S}_{8}$ (1168.2 $\left.\mathrm{g} \mathrm{mol}^{-1}\right): \mathrm{C}, 45.2 ; \mathrm{H}, 5.5 ; \mathrm{S}, 22.0$. Found: $\mathrm{C}, 45.6 ; \mathrm{H}, 5.8 ; \mathrm{S}, 22.6 \%$.

Bis- $\{$ bis-[4-methoxyphenyl(2-

propyl)dithiophosphinato]zinc(II) $\}$

$\left[\mathrm{Zn}\left(\mu-\mathrm{DTFA}^{4}\right)_{2}\left(\mathrm{DTFA}^{4}\right)_{2}\right]$. Yield: $2.00 \mathrm{~g}(81 \%)$. Colorless. M.P. $209-210^{\circ} \mathrm{C} .{ }^{1} \mathrm{H}$ NMR $\left(\mathrm{CDCl}_{3}\right): \delta$ $=1.16\left(\mathrm{dd},{ }^{3} \mathrm{~J}_{\mathrm{PH}}=21.5 \mathrm{~Hz},{ }^{3} \mathrm{~J}_{\mathrm{HH}}=6.9 \mathrm{~Hz}, 12 \mathrm{H}\right.$, $\left.\mathrm{CH}-\left(\mathrm{C}_{3}\right)_{2}\right), 2.3\left(\mathrm{~m}, 4 \mathrm{H},-\underline{\mathrm{H}}^{-}\right), 3.84(\mathrm{~s}, 6 \mathrm{H}$, $\left.\mathrm{OCH}_{3}\right), 6.94\left(\right.$ A-part of $\mathrm{AA}^{\prime} \mathrm{MM}^{\prime} \mathrm{X},{ }^{4} \mathrm{~J}_{\mathrm{PH}}=2.35 \mathrm{~Hz}$ $\left.\left(J_{A X}\right), N=8.80 \mathrm{~Hz}, 4 \mathrm{H}, m-\mathrm{H}\right), 7.83 \mathrm{M}$-part of $\mathrm{AA}^{\prime} \mathrm{MM}^{\prime} \mathrm{X},{ }^{3} \mathrm{~J}_{\mathrm{PH}}=12.95 \mathrm{~Hz}\left(J_{\mathrm{MX}}\right), N=8.80 \mathrm{~Hz}, 4 \mathrm{H}$, $\mathrm{o}-\mathrm{H}) .{ }^{13} \mathrm{C}-\mathrm{NMR}\left(\mathrm{ppm}, \mathrm{CDCl}_{3}\right): \delta=16.26\left(\mathrm{~d},{ }^{2} \mathrm{~J}_{\mathrm{P}-\mathrm{C}}\right.$ $\left.=1.0 \mathrm{~Hz},-\left(\mathrm{CH}_{3}\right)_{2}\right), 38.19\left(\mathrm{~d}, \mathrm{~J}_{\mathrm{P}-\mathrm{C}}=49.6 \mathrm{~Hz}, \mathrm{P}-\right.$ $\underline{\mathrm{CH}}-), 55.37\left(\mathrm{~s}, \underline{\mathrm{CH}}_{3} \mathrm{O}-\right), 113.6\left(\mathrm{~d},{ }^{3} \mathrm{~J}_{\mathrm{P}-\mathrm{C}}=13.9\right.$ $\left.\mathrm{Hz}, \operatorname{Ar}-\mathrm{C}_{\text {meta }}\right), \delta=124.85\left(\mathrm{~d}, \mathrm{~J}_{\mathrm{P}-\mathrm{C}}=78.1 \mathrm{~Hz}, \mathrm{P}-\right.$ Carom) $132.97\left(\mathrm{~d},{ }^{2} \mathrm{~J}_{\mathrm{P}-\mathrm{C}}=12.5 \mathrm{~Hz}\right.$, Ar-Cortho $)$, $162.05\left(\mathrm{~d},{ }^{4} \mathrm{~J}_{\mathrm{P}-\mathrm{C}}=2.8 \mathrm{~Hz}, \mathrm{CH}_{3} \mathrm{O}-\underline{\mathrm{C}}\right)$. ${ }^{31 \mathrm{P}-\mathrm{NMR}}$ $\left(\mathrm{ppm}, \mathrm{CDCl}_{3}\right): \delta=82.9$. LC/MS: $\mathrm{m} / \mathrm{z} 1133.8$ $\left([\mathrm{M}+\mathrm{Na}]^{+}, 31.2 \%\right) ; 864.9\left(\left[\left(\mathrm{M}-\left(\mathrm{Zn}\left(\mathrm{DTFA}^{4}\right)\right]^{+}\right.\right.\right.$, $\left.100.0 \%) ; 576.9\left([\mathrm{M} / 2]^{+}+\mathrm{Na}\right]^{+}, 78,5 \%\right) ; 308.8$ $\left(\left[\mathrm{Zn}\left(\mathrm{DTFA}^{4}\right]^{+}, 12,3 \%\right)\right.$. Anal. Calcd. for $\mathrm{C}_{40} \mathrm{H}_{56} \mathrm{Zn}_{2} \mathrm{O}_{4} \mathrm{P}_{4} \mathrm{~S}_{8}\left(1112.1 \mathrm{~g} \mathrm{~mol}^{-1}\right): \mathrm{C}, 43.2 ; \mathrm{H}$, $5.1 ; \mathrm{S}, 23.1$. Found: C, 43.2; H, 4.9; S, $23.3 \%$.

\section{RESULT AND DISCUSSION}

The data relating to the percent yields, physical appearance, and elemental analyses of the complexes as well as IR-, mass- and NMR $\left({ }^{1} \mathrm{H}-\right.$, ${ }^{13} \mathrm{C}-$, $\left.{ }^{31} \mathrm{P}-\right)$ spectra are given in the section "Experimental". The major spectroscopic features are as follows:

\section{IR Spectra}

The prominent IR bands of the complexes, $\left[\mathrm{Zn}\left(\mu-\mathrm{DTPA}^{1}\right)_{2}\left(\mathrm{DTPA}^{1}\right)_{2}\right], \quad[\mathrm{Zn}(\mu-$ DTPA $\left.\left.^{2}\right)_{2}\left(\text { DTPA }^{2}\right)_{2}\right], \quad\left[Z n\left(\mu-D_{T P A}^{3}\right)_{2}\left(D^{2} P^{3}\right)_{2}\right]$ and $\left[\mathrm{Zn}\left(\mu-\mathrm{DTPA}^{4}\right)_{2}\left(\mathrm{DTPA}^{4}\right)_{2}\right]$, are summarized in Table 1 . The asymmetrical and symmetrical PS stretching vibrations, vasym and $v_{\text {sym }}$, show up in the ranges $610-588 \mathrm{~cm}^{-1}$ and $492-549 \mathrm{~cm}^{-1}$, respectively. The bands in the range 282-311 $\mathrm{cm}^{-1}$ were assigned to $\mathrm{Zn}-\mathrm{S}$ stretching bands, vzn-s. These values agree well with those reported in the literature for analogous compounds (22-24). 
Table 1: Selected FTIR (R) data $\left(\mathrm{cm}^{-1}\right)$ assignment of significant bands for $\left[\mathrm{Zn}\left(\mu-\mathrm{DTPA}^{\mathrm{n}}\right)_{2}\left(\mathrm{DTPA}^{\mathrm{n}}\right)_{2}\right]$

\begin{tabular}{|c|c|c|c|}
\hline \multirow{2}{*}{ Compounds } & $v_{z n-S}$ & $v_{\text {asym }}$ (PS) & $v_{\text {sym }}$ (PS) \\
\hline & IR & IR & IR \\
\hline$\left[Z n\left(\mu-D T F A^{1}\right)_{2}\left(D_{T F A}{ }^{1}\right)_{2}\right]$ & 308 & $610 ; 599$ & $518 ; 534$ \\
\hline$\left[Z n\left(\mu-D T F A^{2}\right)_{2}\left(D_{T F A}^{2}\right)_{2}\right]$ & 282 & 589 & $517 ; 542$ \\
\hline$\left[\mathrm{Zn}\left(\mu-\mathrm{DTFA}^{3}\right)_{2}\left(\mathrm{DTFA}^{3}\right)_{2}\right]$ & 297 & 588 & 492 \\
\hline$\left[\mathrm{Zn}\left(\mu-\mathrm{DTFA}^{4}\right)_{2}\left(\mathrm{DTFA}^{4}\right)_{2}\right]$ & 311 & 611 & $523 ; 550$ \\
\hline
\end{tabular}

\section{Mass Spectra}

The mass signals of the complexes reflect the isotopical multitudes of zinc and sulfur. Some of the mass peaks indicate the attachment of an $\mathrm{CH}_{3} \mathrm{CN}$ or a $\mathrm{Na}^{+}$cation to the species responsible for the signal. $\mathrm{CH}_{3} \mathrm{CN}$ or $\mathrm{Na}^{+}$ions are likely to be originated from the buffer solution used in the ionization chamber and similar cases were reported in the literature (25). The disintegration pattern of the complexes are also comparable to the literature data reported for analogous structures (26-29). Molecular peaks corresponding to the dimeric structures of the complexes are discernable for all the compounds, but $\left[\mathrm{Zn}\left(\mu-\mathrm{DTPA}^{1}\right)_{2}\left(\mathrm{DTPA}^{1}\right)_{2}\right]$. The complexes display Mass-peaks corresponding to the mono-nuclear [ $\left.\mathrm{Zn}\left(\mathrm{DTPA}^{\mathrm{n}}\right)_{2}\right]$ moieties. In the Mass spectrum of all the $[\mathrm{Zn}(\mu-$ $\left.\mathrm{DTPA}^{\mathrm{n}}\right)_{2}\left(\mathrm{DTPA}^{\mathrm{n}}\right)_{2}$ ] complexes display peaks indicating removal of a DTPA ${ }^{n}$ leaving back a sort of the formula $\left[\mathrm{Zn}_{2}\left(\mathrm{DTPA}^{\mathrm{n}}\right)_{3}\right]$.

\section{${ }^{1}$ H-NMR Spectra}

${ }^{1} \mathrm{H}-\mathrm{NMR}$ data are summarized in Table 2 . For all the structures investigated the four anisole-ring protons and the phosphorus atom constitute an $A^{\prime}{ }^{\prime} M M^{\prime} X$ system. The $A A^{\prime} M M^{\prime}$ part display practically an AMX pattern (30). This is obviously because $J_{\mathrm{AM}^{\prime}}$ and $J_{A^{\prime} M}$ are virtually zero. The aromatic protons were assigned on the basis of the magnitudes of the coupling constants to phosphorus. In all the compounds, the protons in ortho-position to phosphorus display a ${ }^{3} J_{\mathrm{PH}}$ of $\sim 13 \mathrm{~Hz}$ and those meta- to phosphorus a ${ }^{4} J_{\mathrm{PH}}$ of $\sim 2.3 \mathrm{~Hz}$.

The peak area integrals of all the signals confirm the assignments of the peaks. Chemical shift and coupling data are in compliance with the literature $(31,32)$.

\section{${ }^{13}$ C-NMR Spectra}

${ }^{13} \mathrm{C}-\mathrm{NMR}$ data are given in Table 3 . The contact ${ }^{31} \mathrm{P}-{ }^{13} \mathrm{C}$ coupling of the ipso- carbons varies between $76.3 \mathrm{~Hz}$ and $88.8 \mathrm{~Hz}$ whereas the onebond coupling for the aliphatic carbons, C6, lies in the range $48.1 \mathrm{~Hz}-51.4 \mathrm{~Hz}$. The $\delta$ and $J$ values are in agreement with those given in the literature (33-35).

\section{${ }^{31}$ P-NMR Spectra}

Proton-decoupled ${ }^{31} \mathrm{P}-\mathrm{NMR}$ signals of the compounds, $\left[\mathrm{Zn}\left(\mu-\mathrm{DTPA}^{1}\right)_{2}\left(\mathrm{DTPA}^{1}\right)_{2}\right], \quad[\mathrm{Zn}(\mu-$ $\left.\left.\mathrm{DTPA}^{2}\right)_{2}\left(\mathrm{DTPA}^{2}\right)_{2}\right], \quad\left[\mathrm{Zn}\left(\mu-\mathrm{DTPA}^{3}\right)_{2}\left(\mathrm{DTPA}^{3}\right)_{2}\right]$ and $\left[\mathrm{Zn}\left(\mu-\mathrm{DTPA}^{4}\right)_{2}\left(\mathrm{DTPA}^{4}\right)_{2}\right]$, appear at $68.1,60.1$, 79.4 and 82.9 ppm, respectively. The fact that each compound displays only one singlet indicate that the phosphorus atoms are in an equivalent environment within the molecule except for $\left[\mathrm{Zn}\left(\mu-\mathrm{DTPA}^{2}\right)_{2}\left(\mathrm{DTPA}^{2}\right)_{2}\right]$. These data comply with those reported in the literature (31).

\section{CONCLUSION}

In this work four new dithiophosphinato zinc complexes were synthesized and characterized. The characteristic $v_{\mathrm{N}-\mathrm{H}}$ stretching bands of the ligands appear at $\sim 3000-3100 \mathrm{~cm}^{-1}$ on IR (1820). These bands disappear on the IR spectra of the complexes proving the substitution of the ammonium group by metal cations. All the complexes are of dimeric structures. The onebond ${ }^{13} \mathrm{C}-{ }^{31} \mathrm{P}$ coupling constants for the aromatic ipso- carbons were found to be $30-40 \mathrm{~Hz}$ greater than that of the aliphatic carbons $(76.3-88.8 \mathrm{~Hz}$ and 48.1-51.4 Hz, respectively). Due to the equivalent chemical environment of the phosphorus in the complexes except for $[\mathrm{Zn}(\mu-$ DTPA $\left.\left.^{2}\right)_{2}\left(\text { DTPA }^{2}\right)_{2}\right]$, a single peak appeared in the ${ }^{31} \mathrm{P}-\mathrm{NMR}$ spectrum. The structures of the complexes were elucidated on the basis of elemental analysis as well as mas-, FTIR- and NMR-spectra. 


\begin{tabular}{|c|c|c|c|c|c|}
\hline & & & $\overbrace{\mathrm{H}_{3} \mathrm{C} \mathrm{C}}^{1}$ & & \\
\hline $\begin{array}{c}{[\mathrm{Zn}(\mu-} \\
\left.\left.\text { DTFA }^{1}\right)_{2}\left(\mathrm{DTFA}^{\mathbf{1}}\right)_{2}\right] \\
\mathrm{R}^{\mathbf{1}}=\text { iso-amyl- }\end{array}$ & \multicolumn{2}{|c|}{ AA'MM'X spin system, $\left(N=J_{\mathrm{AM}}+J_{\mathrm{AM}^{\prime}}\right)$} & $-\mathrm{OC} \underline{\mathbf{H}}_{3}$ & $-\mathrm{C} 7-\underline{\mathrm{H}}$ & -C9-브 \\
\hline 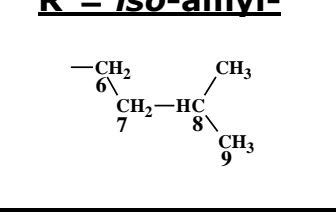 & $\begin{array}{c}\delta=7.86 \\
(d d, 8 H) \\
3 J_{P-H}=13.36,\left(J_{M X}\right) \\
N=8.70\end{array}$ & $\begin{array}{c}\delta=6.95 \\
(d d, 8 H) \\
{ }^{4} J_{P-H}=2.30 \\
\left(J_{A X}\right) \\
N=8.70\end{array}$ & $\begin{array}{l}\delta=3.84 \\
(s, 12 \mathrm{H})\end{array}$ & $\begin{array}{l}\delta=1.55 \\
(m, 8 H)\end{array}$ & $\begin{array}{c}\delta=0.85 \\
(d, 24 \mathrm{H}) \\
3 J_{\mathrm{HH}}= \\
6.51\end{array}$ \\
\hline \multicolumn{6}{|l|}{$\begin{array}{c}{[\mathrm{Zn}(\mu-} \\
\left.\left.\text { DTFA }^{2}\right)_{2}\left(\mathrm{DTFA}^{2}\right)_{2}\right] \\
\mathrm{R}^{2}=\text { iso-butyl- }\end{array}$} \\
\hline 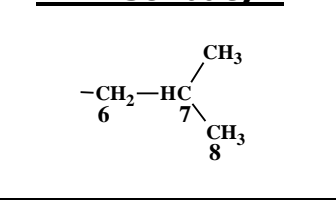 & $\begin{array}{c}\delta=7.91 \\
(\mathrm{dd}, 8 \mathrm{H}) \\
3_{\mathrm{P}-\mathrm{H}}=13.44,\left(J_{\mathrm{MX}}\right) \\
N=8.50\end{array}$ & $\begin{array}{c}\delta=6.92 \\
(\mathrm{dd} 8 \mathrm{H}) \\
{ }^{4} J_{\mathrm{P}-\mathrm{H}}=2.46, \\
\left(J_{\mathrm{AX}}\right) \\
\mathrm{N}=8.50\end{array}$ & $\begin{array}{l}\delta=3.84 \\
(s, 12 \mathrm{H})\end{array}$ & $\begin{array}{c}\delta=2.11 \\
(m, 24 \mathrm{H})\end{array}$ & - \\
\hline \multicolumn{6}{|l|}{$\begin{array}{c}{[\mathrm{Zn}(\mu-} \\
\left.\left.\mathrm{DTFA}^{3}\right)_{2}\left(\mathrm{DTFA}^{3}\right)_{2}\right] \\
\mathrm{R}^{3}=\text { sec- butyl- }^{-}\end{array}$} \\
\hline 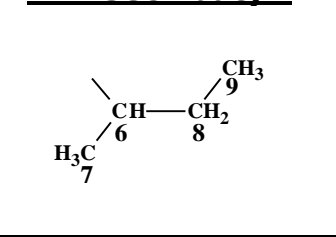 & $\begin{array}{c}\delta=7.83 \\
(\mathrm{dd}, 8 \mathrm{H}) \\
3 J_{\mathrm{P}-\mathrm{H}}=12.94,\left(J_{\mathrm{MX}}\right) \\
\mathrm{N}=8.80\end{array}$ & $\begin{array}{c}\delta=6.92 \\
(\mathrm{dd} 8 \mathrm{H}) \\
{ }^{4} J_{\mathrm{P}-\mathrm{H}}=2.32 \\
\left(J_{\mathrm{AX}}\right) \\
\mathrm{N}=8.80\end{array}$ & $\begin{array}{l}\delta=3.84 \\
(s, 12 \mathrm{H})\end{array}$ & $\begin{array}{c}\delta=1.16 \\
\text { (m, C6-H and } \\
\text { C7-H adjacent, } \\
16 \mathrm{H})\end{array}$ & $\begin{array}{c}\delta=0.92 \\
(t, 12 \mathrm{H}) \\
3 \mathrm{~J}_{\mathrm{HH}}= \\
7.30\end{array}$ \\
\hline
\end{tabular}

$$
[\mathrm{Zn}(\boldsymbol{\mu}-
$$

$\left.\left.\operatorname{DTFA}^{4}\right)_{2}\left(\text { DTFA }^{4}\right)_{2}\right]$

$\mathrm{R}^{4}=$ iso-propyl- 


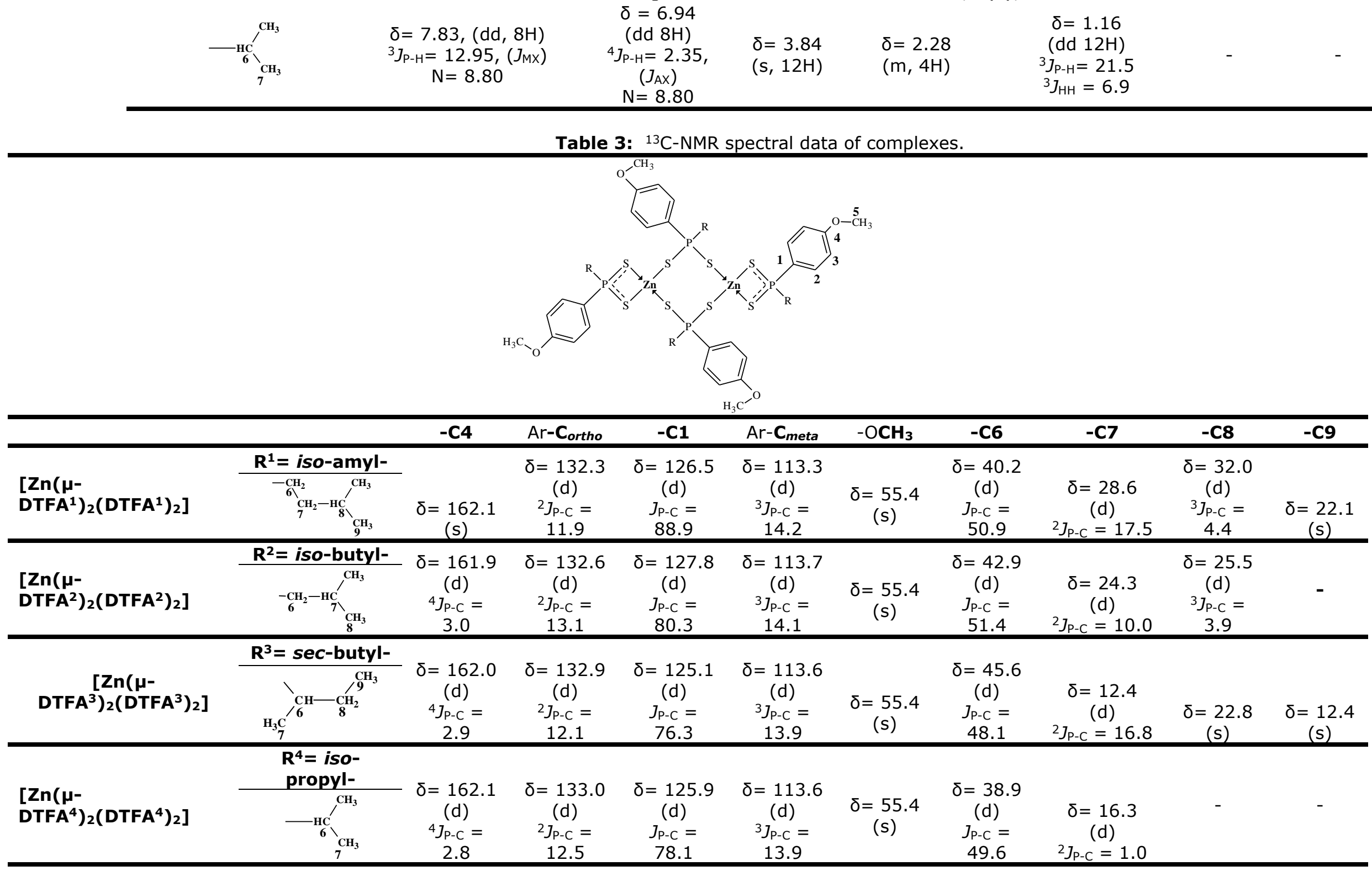

Chemical shifts $(\delta)$ are reported in ppm. $J$ values are reported in Hz. s: singlet; d: doublet; t: triplet; dd: doublet of doublets; m: multiplet. 


\section{ACKNOWLEDGEMENTS}

We gratefully acknowledge the financial assistance of Project Coordination Application and Research Center of Bozok University (Project No: 2013 BAP FEF/A049).

\section{REFERENCES}

1. Mc.Cleverty J A, Rick S Z, Kowalski N, Bailey A, Mulvaney R, O'Cleirigh D A. Aspects of the Inorganic Chemistry of Rubber Vulcanisation. Part 4.1 Dialkyl- and Diaryldithiophosphate and -dithiophosphinate Complexes of Zinc: Phosphorus-31 Nuclear Magnetic Resonance Spectral Studies and Structures of [NMe4][Zn\{S,P(OC6H4Mep $\left.\left.)_{2}\right\}_{3}\right]$ and [Et4] $\left[\mathrm{Zn}\left(\mathrm{S}_{2} \mathrm{PPh}_{2}\right)_{3}\right]$. J. Chem. Soc. Dalton Trans. 1983; 627-34. DOI 10.1039/DT9830000627.

2. Satake $\mathrm{Y}$, Kashiwadate $\mathrm{K}$, Iizuka $\mathrm{Y}$, Kouyama T, Katto T, Shiiki Z. Poly(arylene thioether-ketone) compositions improved in both melt stability and crystallinity. 1989: may 2 ; US 4,826,906.

3. Pradip K G, Deba P P, Neeiotpal S. Di(2methyl- 5-chlorophenyl) dithiophosphinate and its copper(II) derivative as antioxidant lubricating oil additive. Indian J. Chem. Technol. 1995; 2(1): 46-8. http://nopr.niscair.res.in/handle/123456789/ 31090.

4. Ziyatdinova G K, Budnikov G K, Samigullin A I, Gabdullina G T, Sofronov A V, Al'metkina, L. A., Nizamov, I. S., Cherkasov, R. A.: Electrochemical determination of synthetic antioxidants of bisdithiophosphonic acids. J. Anal. Chem. 2010; 65: 1273-1279. DOI: $10.1134 / S 1061934810120129$.

5. Yagishita $\mathrm{K}$, Konishi S. Lubricating oil additive and lubricating oil composition. 2013; US 8,481,467.

6. Grigorieva N A, Pashkov G L, Fleitlikh I Yu, Nikiforova $L$ K, Pleshkov $M$ A. Nickel extraction from sulfate media with Cyanex 301 in the presence of electron donor additives. Hydrometallurgy 2010; 105: 82-8. DOI 10.1016/hydromet.2010.08.001.

7. Xihong $H$, Guoxin $T$, Jing $C$, Linfeng $R$, Characterization of the extracted complexes of trivalent lanthanides with purified cyanex 301 incomparison with trivalent actinide complexes. Dalton Trans. 2014; 43: 1735217357. DOI:10.1039/C4DT02553A.

8. Jing $C$, Meng $W$, Xuegang $L$, Jianchen $W$. Back-end of nuclear fuel cycle in China. Progr. in Nucl. Energy, 2012; 54: 46-8, DOI: $10.1016 / j$. pnucene.2011.09.003
9. Artem'ev A V, Malysheva S F, Gusarova N K, Belogorlova N A, Fedorov S V, Timokhin B V, Smirnov V I, Trofimov B A. Novel Quinine, Lupinine, And Anabasine Derivatives Containing Dithiophosphinate Groups. Chem. Heterocycl. Compd. 2012 Jun; 48(3): 44852. DOI: $10.1007 / \mathrm{s} 10593-012-1013-3$.

10. Michalski J, Potrzebowski M, Lopusinski A. Preparation of S-brom-dithiophosphoric, dithiophosphonic and dithiophosphinic acid derivatives, 1984; US 4,470,933.

11. Kabra V, Mitharwal S, Singh S. Synthesis and Insecticidal Activity of Novel Dithiophosphonates. Phosphorus, Sulfur Silicon Relat. Elem. 2009;184: 2431-42. DOI: $10.1080 / 10426500802487748$

12. Bara A C, Silvestru C, Haiduc I. Antitumor organometallics. I. Activity of some diphenyltin(IV) and diphenylantimony(III) derivatives on in vitro and in vivo Ehrlich ascites tumor. Anticancer Res. 11, 16511656, 1991.

13. Bellande E, Comazzi V, Laine J, Lecayon M, Pasqualini R, Duatti A, Hoffschir D, Synthesis and Biodistribution of Nitrido Technetium-99m Radiopharmaceuticals with Dithiophosphinate Ligands:a Class of Brain Imaging Agents. Nucl. Med. and Biol. 1995; 22(3):315-20. DOI: 10.1016/09698051(94)00107-U.

14. Durckheimer W, Bormann D, Ehlers E, Schrinner E, Heymes R. Cephem Derivates. 1988; US 4758556.

15. Yordanov, N D, Gochev G, Angelova O, Macicek J. EPR Investigations on bis(dithiophosphinato)copper(II) complexes magnetically diluted in the corresponding nickel(II) single crystals and X-ray crystal structure of bis(di-npropyldithiophosphinato)nickel(II).

Polyhedron 1990; 9: 2597-2602. https://doi.org/10.1016/S02775387(00)86834-5.

16. Byrom C, Malik M A, O'Brien P, White A J $P$, Williams D J. Synthesis and $X$-ray single crystal structures of bis(diisobutyldithiophosphinato)cadmium(II) or zinc(II): Potential single-source precursors for II/VI materials. Polyhedron, 2000; 19: 211-15. https://doi.org/10.1016/S02775387(99)00352-6.

17. Calligaris $M$, Nardin G, Ripamonti A. Crystal and Molecular Structure of Zinc(II) and Cobalt(II) Diethyldithiophosphinates, J. 
Chem. Soc. A, 714-22, 1970. DOI: $10.1039 / \mathrm{J} 19700000714$.

18. Sağlam E G, Çelik Ö, Yılmaz H, İde S. Synthesis, spectroscopic characterization and $\mathrm{X}$-ray single crystal structures of trans-Bis [ 4-methoxyphenyl dithiophosphinato]nickel(II) and Bis[4methoxyphenyl(3methylbutyl)dithiophosphinato]cobalt(II) complexes. Transition Met. Chem. 2010; 35: 399-405. DOI:10.1007/s11243-010-9341-6.

19. Sağlam E G, Yılmaz H, Dal H, Hökelek T, Synthesis and Spectroscopic Characterizations of Novel $\mathrm{Ni}$ (II) and $\mathrm{Co}$ (II) Dithiophosphinate Complexes, and X-Ray Studies on the $\mathrm{Ni}$ (II) Complex Phosphorus, Sulfur Silicon Relat. Elem. 2012; (2)187: 213-24.

DOI:

10.1080/10426507.2011.601239.

20. Hoffmann $H$, Schumacher G, Umsetzungen mit p-methoxyphenyl-dithiophosphonsäure-anhydrid, Tetrahedron Lett. 1967; 31:2963-6. DOI:10.1016/500404039(00)90895-5.

21. Diemert K, Kuchen W. "Zur Kenntnis der Organophosphorverbindungen XVII, Dithiophosphinsauren RR'P(S)SH, Ihre Synthese, Derivate und MetallKomlexe", Phosphorus, Sulfur Silicon Relat. Elem. 1977; (2): 131-6. DOI: 10.1080/03086647708077702.

22. Casas J S, García-Tasende M S, Sánchez A, Sordo J, Castellano E E, ZukermanSchpector J. Synthesis, crystal structure and spectroscopik properties of bis (diphenylditihiophosphinato)cadmium(II).

Inorg. Chim. Acta 1994; 219: 115-9. DOI: $10.1016 / 0020-1693(94) 03822-8$.

23. Sunder S, Hanlan $L$, Bernstein J. Resonance Raman Spectra of Metal Complexes of Substituted Dithiophospinic Acids., J. Inorg. Chem. 1975;14: 2012-3, DOI: $10.1021 /$ ic50150a058.

24. Czernuszewicz R, Maslowsky E, Jr Nakamoto K, Infrared and Raman Spectra of Bis(imidotetraphenyldithiodiphosphino-S, $\mathrm{S}^{\prime}$ ) Complexes with $\mathrm{Cu}(\mathrm{II}), \mathrm{Co}(\mathrm{II})$ and $\mathrm{Fe}(\mathrm{II})$, Inorg. Chim. Acta, 1980; 40: 199-202. DOI: 10.1016/S0020-1693(00)92004-8.

25. Chakravarty $M$, Pailloux $S$, Ouizem $S$, Smith K A, Duesler E N, Paine R T, Williams N J, Hancock R D. Synthesis and metal coordination chemistry of (phenyl)(pyridin-2ylmethyl)phosphinodithioic acid, [2$\left.\mathrm{C}_{5} \mathrm{H}_{4} \mathrm{~N}\right] \mathrm{CH}_{2} \mathrm{P}(\mathrm{S})(\mathrm{SH})(\mathrm{Ph})$. 2012: 33: 327-35. DOI: $10.1016 /$ j.poly.2011.11.041.
26. Keck $H$, Kuchen W. Massenspektrometrische Untersuchungen An Organophosphorverbindungen IV. Über den massenspektrometrischen Zerfall von Dithiophosphinsären, Phosphorus, Sulfur Silicon Relat. Elem., 1983; 14: 225-8. DOI: $10.1080 / 03086648308075944$.

27. Heinz $S$, Keck $H$, Kuchen W. Mass spectrometric studies of dithiophosphinato metal complexes. Org. Mass Spoctrom. 1984; 19: 82-6.DOI:10.1002/oms.120190207.

28. Christoph D, Keck H, Kuchen W, Mathow J, Wunderlich $H$. Synthesis, properties and structure bis(dialkyldithiophosphinato)manganese(II) complexes. Inorg. Chim. Acta 1987; 132,: 213-5. DOI:10.1016/S0020-1693(00)817442.

29. Mohan P N, Keck H, Kuchen W, Haegele G. Metal complexes of phosphinic acids-XII: Praseodymium(III), neodymium(III) and europium(III) complexes of dimethyldithiophosphinic acid. J. Inorg. \& Nuclear Chem. 1977; 39: 833-5. DOI: $10.1016 / 0022-1902(77) 80164-4$.

30. Sağlam E G, Nurcan A, Berline M-S, Hakan D, Tuncer H. Syntheses and Structural Characterizations of a New Benzyl(4Methoxyphenyl)Dithiophosphinic Acid and Its $\mathrm{Ni}(\mathrm{II}), \mathrm{Co}(\mathrm{II}), \mathrm{Zn}(\mathrm{II}), \mathrm{Cd}(\mathrm{II})$ and Ni-Pyridino Complexes. Phosphorus, Sulfur Silicon Relat. Elem. 2016; 191(1): 22-9. DOI; $10.1080 / 10426507.2015 .1067206$.

31. Karakuş M, Yılmaz H, Bulak E, Lonnecke, P. Bis $\{\mu-[\mathrm{O}$-cyclopentyl(4-methoxyphenyl) dithiophosphonato]1K: S,2k: S-[Ocyclopentyl (4- methoxyphenyl) dithiophosphonato] $1 \mathrm{k} 2 \mathrm{~S}, \mathrm{~S}\}$ dizinc (II). Appl. Organometal. Chem. 2005; 19: 396-7. DOI: $10.1002 /$ aoc. 850 .

32. Karakuş $M$, Yılmaz $H$, Özcan $Y, S$ İde. Appl. Crystallographic report: Bis $\{\mu-[0-$ dithiophosphonato] 1K:S,2K: S-[Ocyclopentyl(4-methoxyphenyl) dithiophosphonato]-1k2S, $\left.\mathrm{S}^{\prime}\right\}$ dicadmium(II). Organometal. Chem. 2004; 18: 141142.DOI: $10.1002 /$ aoc. 588.

33. Ramirez R G, Toscano R A, Silverstu C, Haiduc I. Studies on inorganic tin diphenyldithiophosphinates. Crystal and molecular structure of CISdichlorobis(diphenyldithiophosphinato)tin(IV). Polyhedron 1996; 15(21): 385767.DOI:10.1016/0277-5387(96)00100-3. 
34. Karakuş M, Yılmaz $H$. Synthesis and Characterization of $\mathrm{Ni}(\mathrm{II}), \mathrm{Zn}(\mathrm{II})$, and $\mathrm{Cd}(\mathrm{II})$ Complexes with Dithiophosphonate Derivatives. Rus. J. Coord Chem 2006; 32(6): 437-43. $10.1134 / \mathrm{S} 1070328406060078$.
35. Przychodzen W. New Products of Reaction of Lawesson's Reagent With Diols. Phosphorus, Sulfur Silicon Relat. Elem. 2004; 179:

1621-33. 
